\title{
Pirarubicin reduces USP22 expression by inhibiting CREB-1 phosphorylation in HeLa cells
}

\author{
XIAOOU ZHOU ${ }^{1 *}$, LIJUN GAN $^{2 *}$, JIANYUN LIU $^{3}$, XIN XIE $^{1}$, TAO WANG $^{1}$ and JIANJUN XIONG ${ }^{1,3}$ \\ ${ }^{1}$ Department of Pharmacology, College of Basic Medical Science; ${ }^{2}$ Department of Clinical Nursing, College of Nursing, \\ Jiujiang University; ${ }^{3}$ Key Laboratory of Jiangxi Province for The Systems Biomedicine, Jiujiang, Jiangxi 332000, P.R. China
}

Received May 16, 2018; Accepted November 2, 2018

DOI: $10.3892 /$ etm.2019.7447

\begin{abstract}
The expression of ubiquitin specific peptidase 22 (USP22) is upregulated in several types of cancer, and has been implicated in tumorigenesis. Pirarubicin (THP), an anthracycline antineoplastic drug, can induce apoptosis of several types of cancer cells. However, the molecular mechanisms underlying the action of THP remain to be elucidated. In the current study, treatment with THP induced HeLa cell apoptosis and decreased USP22 expression in a dose- and time-dependent manner. THP reduced the USP22 promoter-regulated luciferase activity, regardless of the mutation of transcriptional activator MYB or E3 ubiquitin-protein ligase SP1 binding sequences; however, this effect was abrogated by the mutation of cyclic AMP-responsive element-binding protein (CREB) binding sequence in HeLa cells. Furthermore, the inhibition on the USP22 promoter activity by THP was not affected by overexpression of CREB-1 in HeLa cells. Additionally, treatment with THP significantly decreased the phosphorylation of CREB-1 at ser133 in HeLa cells. Quantitative chromatin immunoprecipitation assay revealed that THP significantly inhibited the binding of CREB-1 to the USP22 promoter in HeLa cells. The present study demonstrated that THP decreased USP22 expression and promoted HeLa cell apoptosis partially by inhibiting the phosphorylation of CREB-1. The current results may provide novel insights into the molecular mechanisms underlying the pharmacological effect of THP on cancer cell apoptosis.
\end{abstract}

Correspondence to: Dr Tao Wang or Dr Jianjun Xiong, Department of Pharmacology, College of Basic Medical Science, Jiujiang University, 17 Lufeng Road, Jiujiang, Jiangxi 332000, P.R. China

E-mail: comwangtaocom@163.com

E-mail: xiongjj1975@163.com

*Contributed equally

Key words: pirarubicin, ubiquitin specific peptidase 22, cyclic AMP-responsive element-binding protein-1, phosphorylation, HeLa

\section{Introduction}

Pirarubicin (THP) is an antitumor drug commonly used for treatment of several types of cancer, with fewer toxic cardiovascular effects compared with its analogue doxorubicin $(1,2)$. THP intercalates into DNA and interacts with topoisomerase II to inhibit DNA replication and promote cancer cell apoptosis (3). A previous study indicated that treatment with a low dose of THP induces MG-63 cell cycle arrest and apoptosis by decreasing the expression of proliferating cell nuclear antigen, cyclin D1, cyclin E and apoptosis regulator Bcl-2, and increasing the expression of apoptosis regulator Bax (4). Furthermore, THP inhibits the expression of cyclin B1 and phosphorylation of cyclin-dependent kinase 1 (Cdc2) in multidrug-resistant osteosarcoma cells (5). In addition, THP induces autophagy in bladder cancer cells (6). However, the molecular mechanisms underlying the effect of THP on cervical cancer cell apoptosis remain to be elucidated.

Ubiquitin-specific peptidase 22 (USP22) is a ubiquitin hydrolase containing a zinc-finger domain at the N-terminus and a ubiquitin-specific peptidase domain at the $\mathrm{C}$ terminus (7). USP22 acts as a subunit of the SAGA transcriptional complex and deubiquitylates histones $\mathrm{H} 2 \mathrm{~A}$ and $\mathrm{H} 2 \mathrm{~B}$ to promote gene transcription $(8,9)$. USP22 may co-activate the Myc proto-oncogene protein or cellular tumor antigen p53-driven target gene transcription (10). Furthermore, USP22 interacts with non-histone substrates and its deubiquitinase activity leads to the stabilization of cyclooxygenase-2, sirtuin 1 , fructose-bisphosphatase 1 and cyclin B1 (11-14). Therefore, USP22 serves a number of roles in the regulation of cell proliferation, cell cycle and apoptosis and may promote tumorigenesis. Upregulated expression of USP22 was previously detected in several types of cancer, including lung and colon cancer, and is associated with tumor recurrence, metastasis and poor survival of patients with cancer $(15,16)$. By contrast, the knockdown of USP22 leads to cell cycle arrest and reduces cell viability (10). Increased expression of USP22 among patients with cancer is associated with decreased survival rates and this gene may serve as a target for cancer therapy (17). In our previous study, the 3.0-kb USP22 promoter was cloned to identify the basic activity region containing motifs for the binding of cyclic AMP-responsive element-binding protein (CREB), transcriptional activator MYB and E3 ubiquitin-protein ligase SP1 (SP1), regulating the 
USP22 promoter activity (18). Previous studies indicated that chemotherapeutic drug cisplatin (19) or histone deacetylase inhibitor trichostatin A (TSA) (20) induce cancer cell apoptosis by inhibiting the expression of USP22. THP induces cancer cell apoptosis by modulating the expression of several regulators of proliferation and apoptosis (5). The current study hypothesized that THP may directly or indirectly downregulate the expression of USP22 through modulating the expression or activity of transcription factors and promote cancer cell apoptosis.

Human cervical cancer HeLa cells were used in the present study to determine the effect of THP on apoptosis and the expression levels of USP22, and to elucidate the underlying mechanisms. The results indicated that THP induced HeLa cell apoptosis and decreased the transcription of USP22 by inhibiting CREB-1 phosphorylation and binding to the USP22 promoter. These results may provide novel insights into the molecular mechanisms underlying the pharmacological action of THP in inducing cancer cell apoptosis.

\section{Materials and methods}

Cell culture. Human cervical cancer HeLa cells were obtained from the Shanghai Cell Bank of Chinese Academy of Sciences (Shanghai, China) and cultured in Dulbecco's modified Eagle's medium supplemented with $10 \%$ fetal bovine serum (both Gibco; Thermo Fisher Scientific, Inc., Waltham, MA, USA), $100 \mathrm{U} / \mathrm{ml}$ penicillin and $100 \mu \mathrm{g} / \mathrm{ml}$ streptomycin at $37^{\circ} \mathrm{C}$ in a humidified atmosphere of $5 \% \mathrm{CO}_{2}$. THP (Sigma-Aldrich; Merck KGaA, Darmstadt, Germany) was initially dissolved in phosphate buffered saline (PBS) and subsequently diluted in the culture medium to the desired concentrations.

Flow cytometry. The effect of THP on apoptosis of HeLa cells was determined by flow cytometry using the Annexin V-FITC Apoptosis Detection kit, according to the manufacturer's protocol (Beyotime Institute of Biotechnology, Haimen, China). Briefly, HeLa cells $\left(1 \times 10^{5}\right.$ cells/well) were cultured in 6-well plates overnight and treated in triplicate with vehicle PBS or THP $(100-1,000 \mathrm{ng} / \mathrm{ml})$ for $24 \mathrm{~h}$ at $37^{\circ} \mathrm{C}$. The cells were washed and stained with Annexin V-FITC/propidium iodide and the percentages of apoptotic cells were examined by flow cytometry using the FACSCalibur platform (BD Biosciences, Franklin Lakes, NJ, USA). The results were analyzed using the CellQuest software, version 5.1 (BD Biosciences).

Reverse transcription-quantitative polymerase chain reaction $(R T-q P C R)$. HeLa cells were treated in triplicate with vehicle or $100-1,000 \mathrm{ng} / \mathrm{ml}$ THP for $24 \mathrm{~h}$ or with vehicle or $500 \mathrm{ng} / \mathrm{ml}$ THP for 3-24 h at $37^{\circ} \mathrm{C}$. Total RNA from cells treated with vehicle or THP was extracted using TRIzol reagent (Thermo Fisher Scientific, Inc.), and reverse transcribed into cDNA using the TIANScript RT kit (Tiangen Biotech Co., Ltd., Beijing, China). The relative levels of USP22 mRNA to GAPDH mRNA transcripts in individual groups of cells were determined by the qPCR using the SYBR Green PCR master mix (Tiangen Biotech Co., Ltd.) and ABI 7500 Real-Time PCR system (Applied Biosystems; Thermo Fisher Scientific, Inc.). The PCR thermocycling conditions were as follows: Initial denaturation at $95^{\circ} \mathrm{C}$ for $5 \mathrm{~min}, 40$ cycles of denaturation at $95^{\circ} \mathrm{C}$ for $30 \mathrm{sec}$, annealing at $60^{\circ} \mathrm{C}$ for $30 \mathrm{sec}$ and elongation at $72^{\circ} \mathrm{C}$ for $30 \mathrm{sec}$, and the final elongation at $72^{\circ} \mathrm{C}$ for $1 \mathrm{~min}$. The following primers were used: Forward, 5'-GTGTCTTCTTCGGCTGTTTA-3' and reverse, 5'-CTCCTCCTTGGCGATTATTT-3'; USP22 (158 bp) forward, 5'-AGAAGGCTGGGGCTCATTTG-3' and reverse, 5'-AGGGGCCATCCACAGTCTTC-3' for GAPDH (258 bp). Data were analyzed using the $2^{-\triangle \Delta C q}$ method (21).

Western blot analysis. HeLa cells were treated in triplicate with vehicle or THP (100-1,000 ng/ml) for $24 \mathrm{~h}$. Cells were washed and lysed in radioimmunoprecipitation assay solution (Beyotime Institute of Biotechnology) containing a cocktail of protease inhibitors (Sigma-Aldrich; Merck KGaA), followed by centrifugation at $12,000 \mathrm{x} \mathrm{g}$ and $4^{\circ} \mathrm{C}$ for $10 \mathrm{~min}$. Protein concentrations in individual lysates were determined using the bicinchoninic acid method. Samples of $30 \mu \mathrm{g}$ protein/lane were separated by SDS-PAGE on $10 \%$ gels and electrophoretically transferred onto polyvinylidene difluoride membranes. The membranes were blocked with 5\% dry skim milk in tris-buffered saline with Tween-20 and incubated with primary antibodies against CREB-1 (1:1,000 dilution; cat. no. sc-374227), phosphorylated CREB-1 (Ser133; 1:500 dilution; cat. no. sc-101663), USP22 (1:1,000 dilution; cat. no. sc-69082) and GAPDH (1:5,000 dilution; cat. no. sc-20358) at $4^{\circ} \mathrm{C}$ overnight (all Santa Cruz Biotechnology, Inc., Dallas, TX, USA). The membranes were subsequently washed and the bound antibodies were detected with horseradish peroxidase-conjugated secondary mouse anti-goat (cat. no. sc-2354) and anti-rabbit (cat. no. sc-2357; both 1:8,000; Santa Cruz Biotechnology, Inc.) antibodies. ECL kit (Beyotime Biotechnology) was used for visualization. The levels of target proteins relative to control GAPDH were determined by densitometric analysis using ImageJ software (version 1.38; National Institutes of Health, Bethesda, MD, USA).

Transfection and dual luciferase assays. The USP22 promoter and its mutant constructs were generated as previously described $(18,19)$. HeLa cells were cultured in 24-well plates and transfected with $0.8 \mu \mathrm{g}$ pGL3-basic construct, P-2828 (-2828/+52), P-595 (-595/+52), or P-210 (-21/+52) promoter regions together with $0.2 \mu \mathrm{g}$ pRL-TK (Promega Corporation, Madison, WI, USA) using Lipofectamine ${ }^{\circledR} 2000$ (Invitrogen; Thermo Fisher Scientific, Inc.), according to the manufacturer's protocol. A total of $24 \mathrm{~h}$ after transfection, cells were treated with $500 \mathrm{ng} / \mathrm{ml}$ THP and cultured for another $24 \mathrm{~h}$ at $37^{\circ} \mathrm{C}$. Subsequently, cells were lysed and used for dual luciferase assays using Dual-Luciferase ${ }^{\circledR}$ Reporter Assay System (Promega Corporation) according to the manufacturer's protocols. In addition, cells were transfected with plasmids: P-210, P-210/MYB mutant (mut), P-210/CREB mut, $\mathrm{P}-210 / \mathrm{SP} 1$ mut $(0.5 \mu \mathrm{g}$ for each), with or without $0.5 \mu \mathrm{g}$ plasmid for CREB-1 expression [pCMV-CREB (Beyotime Institute of Biotechnology)] as previously described (19). The empty vector pCMV-control $(0.5 \mu \mathrm{g})$ was used as the blank group. Following treatment with THP, the cells were lysed and used for dual luciferase assays as described above.

Chromatin immunoprecipitation (ChIP). The impact of treatment with THP on the binding of CREB-1 to the USP22 promoter in HeLa cells was determined using ChIP, as previously described (18). Briefly, HeLa cells (1x107/group) were treated with or without (the black group) $500 \mathrm{ng} / \mathrm{ml}$ THP for $24 \mathrm{~h}$. 
A

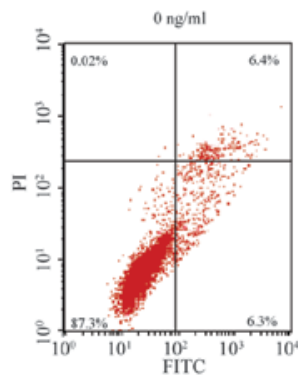

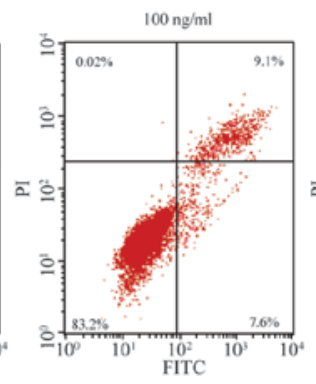
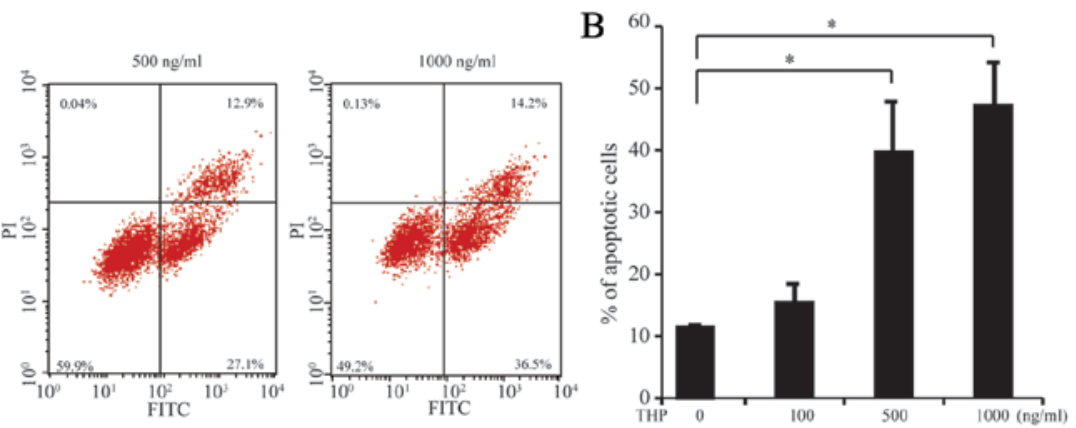

Figure 1. THP induces apoptosis in HeLa cells. HeLa cells were treated with the indicated doses of THP for 24 h, and stained with FITC-Annexin V and PI. The percentages of FITC ${ }^{+}$early stage and FITC $^{+} \mathrm{PI}^{+}$late stage apoptotic cells were determined by flow cytometry. (A) Representative flow cytometry dot plots. (B) Quantitative data presented as the mean \pm standard deviation of each group of cells from three separate experiments. ${ }^{*} \mathrm{P}<0.05$ as indicated. THP, pirarubicin; FITC, fluorescein isothiocyanate; PI, propidium iodide.

Subsequently, the THP-treated or untreated cells were fixed with $1 \%$ formaldehyde and lysed in SDS lysis buffer, followed by sonication and centrifugation at $12,000 \times \mathrm{g}$ and $4^{\circ} \mathrm{C}$ for $10 \mathrm{~min}$. In the THP-treated group, a total of $10 \mathrm{ml}$ supernatant was removed and used as the input sample. Subsequently, cell lysates were incubated with $1 \mu \mathrm{g}$ anti-CREB-1 (cat. no. sc-374227) or negative control immunoglobulin G (cat. no. sc-2025; both Santa Cruz Biotechnology, Inc.) antibodies overnight at $4^{\circ} \mathrm{C}$ and the resulting immunocomplex was purified by magnetic protein-G beads. The immunocomplex was washed, eluted and crosslinked with formaldehyde, followed by treatment with proteinase $\mathrm{K}$ at $67^{\circ} \mathrm{C}$ overnight. The remaining DNA was purified using spin columns and analyzed by PCR using the following primers: forward, CREB 5'-GTC TACCCAGAGCCTAACGG-3' and reverse, 5'-GCGGAGGCC GGACAAAGATGGG-3'. A PCR analysis was conducted to analyze DNA using 2xTaq PCR Mastermix (Tiangen, Inc., China). The procedure used for PCR was as follows: $95^{\circ} \mathrm{C}$ for $5 \mathrm{~min}$, followed by 32 cycles of $95^{\circ} \mathrm{C}$ for $30 \mathrm{sec}, 58^{\circ} \mathrm{C}$ for $30 \mathrm{sec}$ and $72^{\circ} \mathrm{C}$ for $30 \mathrm{sec}$, and the final elongation at $72^{\circ} \mathrm{C}$ for $1 \mathrm{~min}$. The PCR products were resolved by agarose gel electrophoresis on $2 \%$ gel with ethidium bromide and semi-quantitatively analyzed by ImageJ software (version 1.38).

Statistical analysis. Data are presented as the mean \pm standard deviation. The differences between groups were analyzed by one-way analysis of variance followed by Tukey's HSD post-hoc test/Tukey-Kramer method. $\mathrm{P}<0.05$ was considered to indicate a statistically significant difference.

\section{Results}

THP induces apoptosis in HeLa cells. HeLa cells were treated with $0,100,500$ or $1,000 \mathrm{ng} / \mathrm{ml}$ of THP for $24 \mathrm{~h}$, and the percentages of apoptotic cells were determined by flow cytometry. Compared with the vehicle group, treatment with THP at $100 \mathrm{ng} / \mathrm{ml}$ did not significantly alter the percentages of apoptotic HeLa cells (Fig. 1). However, treatment with THP at a dose of 500 and $1,000 \mathrm{ng} / \mathrm{ml}$ significantly increased the percentages of apoptotic cells compared with the vehicle group (both $\mathrm{P}<0.05$ ) and the effect of THP on apoptosis exhibited a dose-dependent trend. The above results indicate that treatment with THP induced HeLa cell apoptosis in vitro.
THP suppresses endogenous USP22 expression. Previous studies indicated that USP22 was associated with antitumor chemotherapy-induced apoptosis (22). The current study tested whether treatment with THP could alter the expression of USP22. HeLa cells were treated with different doses of THP for $24 \mathrm{~h}$ and the relative levels of USP22 mRNA transcripts were determined by RT-qPCR (Fig. 2A). Treatment with $100 \mathrm{ng} / \mathrm{ml}$ THP did not significantly alter the expression levels of USP22 mRNA transcripts; however, treatment with 500 or $1,000 \mathrm{ng} / \mathrm{ml}$ THP significantly decreased the relative levels of USP2 2 mRNA transcripts in HeLa cells (both $\mathrm{P}<0.05$ ). Furthermore, treatment with THP at $500 \mathrm{ng} / \mathrm{ml}$ for $3-12 \mathrm{~h}$ significantly decreased the relative levels of USP22 mRNA transcripts compared with the vehicle group in what appeared to be a time-dependent manner (Fig. 2B). In addition, treatment with THP at 500 or $1,000 \mathrm{ng} / \mathrm{ml}$ markedly reduced the relative protein expression level of USP22 in HeLa cells (Fig. 2C). These results indicate that THP may effectively decrease the expression of USP22 in HeLa cells.

THP decreases the USP22 promoter activity dependent on the CREB motif. To investigate the effect of THP on the USP22 promoter activity, HeLa cells were transfected with the control plasmid pGL3-basic or plasmids with human wild-type USP22 promoter regions P-2828, P-595, or P-210, followed by treatment with vehicle or $500 \mathrm{ng} / \mathrm{ml}$ THP for $24 \mathrm{~h}$. Luciferase activities of individual groups of cells are presented in Fig. 3A. Compared with cells treated with vehicle, treatment with THP significantly reduced the USP22 promoter-driven luciferase activity in all groups (all $\mathrm{P}<0.05$; Fig. 3A). These results indicated that THP inhibited the USP22 promoter activity. Subsequently, HeLa cells were transfected with P-210, P-210/MYB mut, P-210/CREB mut or P-210/SP1 and treated with THP, followed by the luciferase activity measurement in individual groups of cells (Fig. 3B). The results indicated that treatment with THP significantly decreased the P-210-controlled luciferase activity, regardless of the mutation in MYB or SP1 binding sequences in HeLa cells. However, treatment with THP did not alter the P-210/CREB mut-controlled luciferase activity in HeLa cells. Therefore, inhibition of USP22 expression by THP may be dependent on the CREB binding in HeLa cells. The current study further investigated whether CREB-1 over-expression 

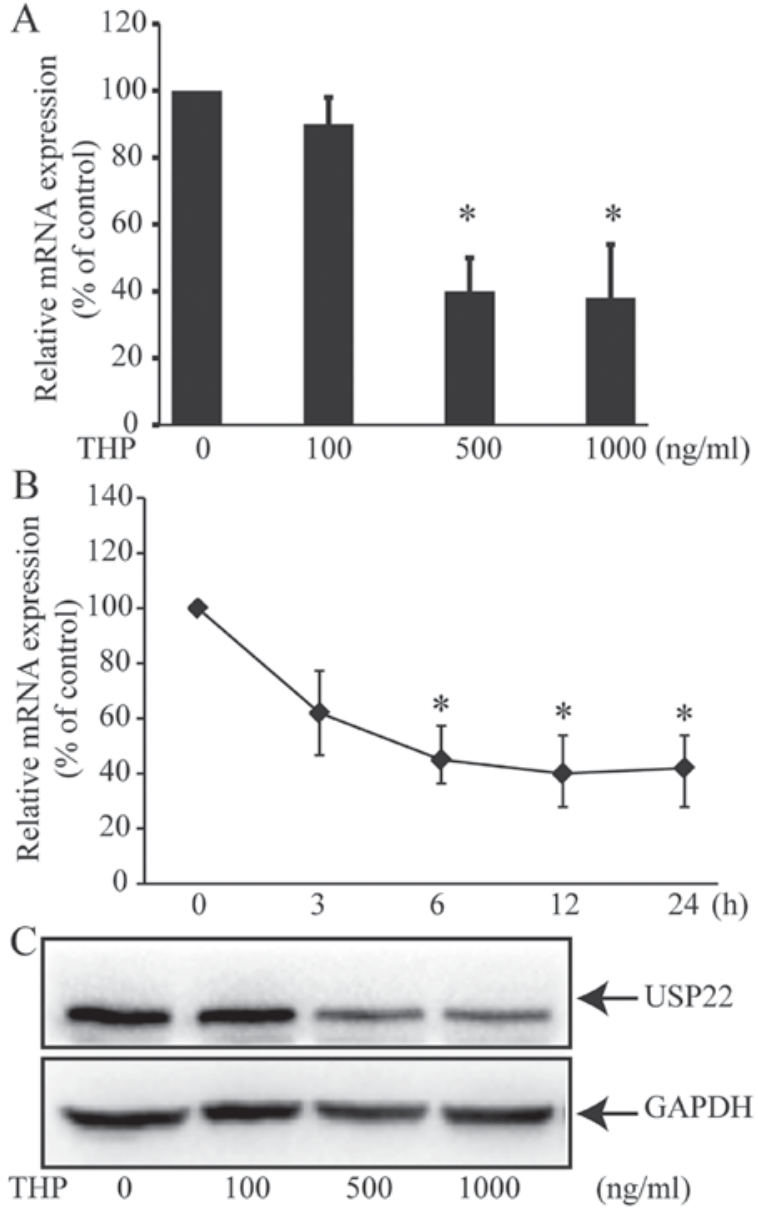

Figure 2. THP decreases USP22 expression in HeLa cells. HeLa cells were treated with different doses of THP for $12 \mathrm{~h}$ or with $500 \mathrm{ng} / \mathrm{ml}$ THP for 0-24 h. The relative levels of USP22 mRNA transcripts were determined by reverse transcription-quantitative polymerase chain reaction or western blotting. (A) THP decreases USP22 expression in a dose-dependent manner. (B) THP decreases USP22 expression compared with the vehicle group in what appears to be a time-dependent manner in. ${ }^{*} \mathrm{P}<0.05$ vs. the vehicle group. (C) Western blot analysis of USP22 expression. Representative images or data presented as the mean \pm standard deviation of each group from three separate experiments are included. ${ }^{~} \mathrm{P}<0.05$ vs. $0 \mathrm{ng} / \mathrm{ml}$ THP. USP22, ubiquitin specific peptidase 22; THP, pirarubicin.

could attenuate the THP-decreased USP22 promoter activity. HeLa cells were co-transfected with P-210 and plasmid for CREB-1 expression for $24 \mathrm{~h}$, and treated with THP. Overexpression of exogenous CREB did not alter P-210 WT promoter activity and the THP-decreased USP22 promoter activity in HeLa cells (Fig. 3C and D).

Treatment with THP decreases CREB phosphorylation. Phosphorylation of CREB is required for its transcriptional activity (23). The present study used western blotting to investigate whether THP could alter the phosphorylation of CREB to decrease the expression of USP22 in HeLa cells. Treatment with 100,500 or $1,000 \mathrm{ng} / \mathrm{ml}$ THP for $12 \mathrm{~h}$ did not significantly alter total CREB-1 protein expression in HeLa cells (Fig. 4). However, treatment with 500 or $1,000 \mathrm{ng} / \mathrm{ml}$ THP significantly decreased the levels of phosphorylated CREB-1 (Ser133) in HeLa cells. These results indicate that THP decreased the USP22 expression by inhibiting the CREB-1 phosphorylation in HeLa cells.

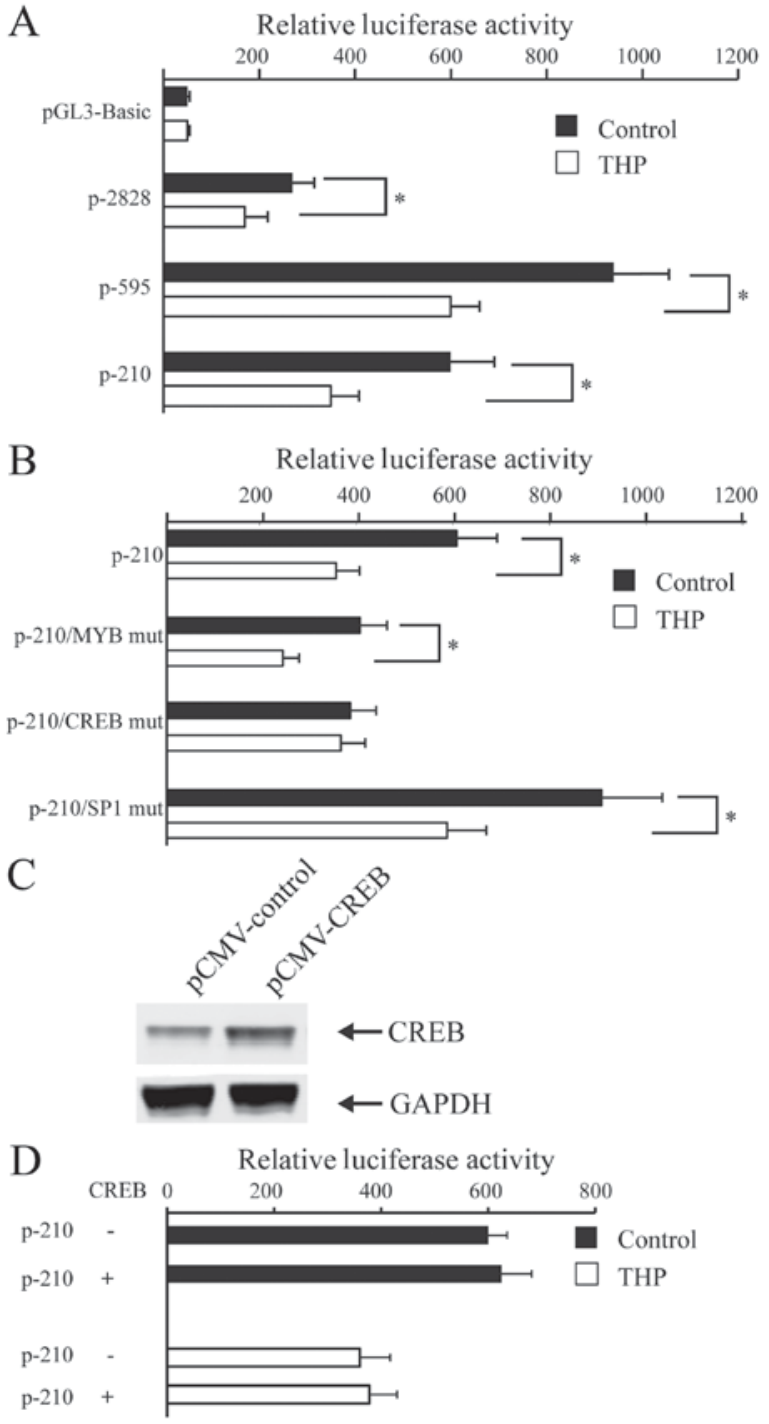

Figure 3. THP inhibits the USP22 promoter activity partially dependent on the binding sequence of CREB in HeLa cells. (A) HeLa cells were transfected with the indicated plasmids for the USP22 promoter-controlled luciferase expression and treated with, or without, $500 \mathrm{ng} / \mathrm{ml}$ of THP. The luciferase activity in individual groups of cells was determined. (B) HeLa cells were transfected with P-210/MYB mut, P-210/CREB mut and P-210/SP1 mut, and treated with, or without, $500 \mathrm{ng} / \mathrm{ml}$ of THP. The luciferase activity in individual groups of cells was determined. " $\mathrm{P}<0.05$ vs. the control group. (C) HeLa cells were transfected with CREB-1 overexpression plasmids and pCMV plasmids, which were used as the blank control. (D) CREB-1 overexpression did not rescue the THP-decreased USP22 promoter activity. HeLa and CREB-1 overexpressing HeLa cells were treated with, or without, THP and the luciferase activity of individual groups of cells was determined. Representative images or data presented as the mean \pm standard deviation of each group from three separate experiments are included. USP22, ubiquitin specific peptidase 22; CREB, cyclic AMP-responsive element-binding protein-1; THP, pirarubicin; MYB, transcriptional activator MYB; SP1, E3 ubiquitin-protein ligase SP1; mut, mutant.

THP inhibits CREB binding to the USP22 promoter. Our previous study indicated that CREB-1 directly binds to the CREB binding sequence of the basic promoter region of USP22 (24). The present study used ChIP assays with anti-CREB-1 antibody to investigate whether THP could modulate CREB-1 binding to the USP22 promoter. Treatment with THP significantly decreased the level of USP22 promoter DNA bound by anti-CREB-1 antibody in 


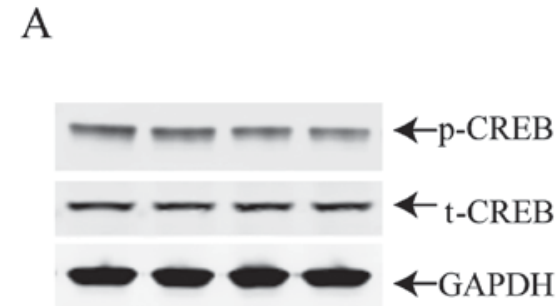

THP $0 \quad 100 \quad 500 \quad 1000(\mathrm{ng} / \mathrm{ml})$

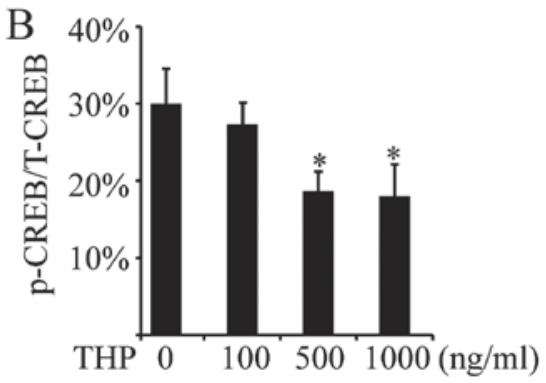

Figure 4. THP decreases CREB phosphorylation in HeLa cells. HeLa cells were treated with the indicated doses of THP for $24 \mathrm{~h}$ and the relative levels of CREB expression and phosphorylation at ser133 were determined by western blotting. (A) Western blot analysis. (B) Quantitative analysis of the ratios of p-CREB to t-CREB in individual groups of cells. Representative images or data presented as the mean \pm standard deviation of each group from three separate experiments are included. " $\mathrm{P}<0.05$ vs. $0 \mathrm{ng} / \mathrm{ml}$ THP. CREB, cyclic AMP-responsive element-binding protein-1; THP, pirarubicin; p, phosphorylated; t, total.
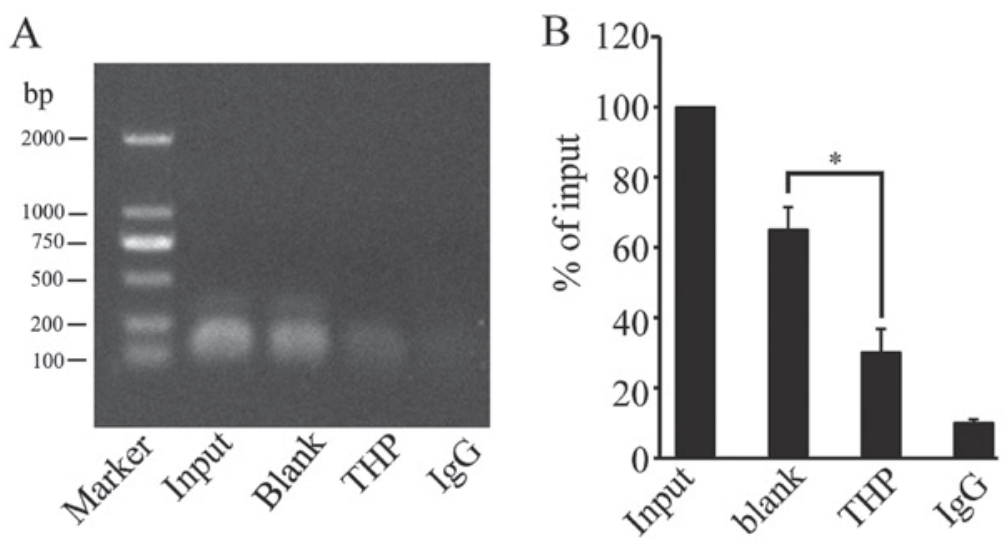

Figure 5. THP inhibits the binding of CREB to the USP22 promoter in HeLa cells. HeLa cells were treated with vehicle or $500 \mathrm{ng} / \mathrm{ml} \mathrm{THP}$ for $3 \mathrm{~h}$, lysed, sonicated and cross-linked. The DNA/protein complexes were immunoprecipitated with control IgG or anti-CREB, followed by polymerase chain reaction amplification of the targeted DNA fragment using specific primers for the USP22 promoter region. Whole cell lysates served as the input control. (A) A representative image. (B) Quantitative analysis. Representative images or data presented as the mean $\% \pm$ standard deviation of each group from three separate experiments are included. "P $<0.05$ as indicated. USP22, ubiquitin specific peptidase 22; CREB, cyclic AMP-responsive element-binding protein-1; THP, pirarubicin; Ig, immunoglobulin.

HeLa cells (Fig. 5). These results demonstrated that treatment with THP may decrease the binding of CREB-1 to the promoter region of USP22 and inhibit the USP22 promoter activity.

\section{Discussion}

Previous studies indicated that treatment with THP can trigger apoptosis of human osteosarcoma (5) and hepatocellular carcinoma (25) cells, and induce autophagy of human cervical cancer cells (6). In the current study, treatment with THP induced cervical cancer cell apoptosis in vitro. The results of the current study support previous observations and indicate that THP may exhibit potent toxicity against numerous types of malignancies.

Upregulated USP22 expression is associated with tumor progression and oncogenesis, while USP22 silencing can induce cell cycle arrest to inhibit growth in several types of tumors $(26,27)$. Furthermore, extracellular stimuli can activate $\mathrm{T}$ and $\mathrm{B}$ lymphocytes and upregulate the expression of USP22 (28). Our previous study revealed that transcription factors SP1 and CREB-1 bound to the USP22 promoter and regulated the expression of USP22 (18). In addition, certain chemotherapeutic drugs, including cisplatin and TSA, downregulate the expression of USP22 and trigger apoptosis in HeLa cells $(19,20)$. In the current study, treatment with THP decreased the expression of USP22 in HeLa cells in a dose- and time-dependent manner. These results support previous observations and indicate that treatment with THP may downregulated the expression of oncogenic and proliferation-associated factors in tumor cells $(4,5)$.

THP is an anthracycline antineoplastic drug inhibiting DNA synthesis in tumor cells (3). THP decreases microRNA-21 expression, stabilizes autophagy related 4B cysteine peptidase mRNA and modulates the phosphorylation of serine/threonine-protein kinase mTOR, ribosomal protein S6 kinase beta-1, eukaryotic translation initiation factor 4E-binding protein 1 and $\mathrm{Cdc} 2$ in different types of tumor cells $(6,25,29)$. In the current study, treatment with THP reduced the USP22 promoter activity in HeLa cells regardless of the presence or absence of the binding sequence of MYB or SP1. However, the inhibitory effect of THP on USP22 expression was dependent on the presence of the CREB binding sequence in the USP22 promoter. Therefore, binding of CREB-1 to the USP22 promoter may be required for THP-mediated inhibition of USP22 expression in HeLa cells. Overexpression of CREB in the present study did not to rescue the THP-mediated inhibition of USP22 promoter-controlled luciferase activity in 
HeLa cells. The present study demonstrated that treatment with THP significantly reduced the phosphorylation of CREB-1 at Ser 133 and the binding of CREB-1 to the USP22 promoter in HeLa cells. Previous studies suggested that the protein kinase A (PKA), protein kinase $\mathrm{B}$ or mitogen activated kinase signaling is necessary for the activation of CREB and its binding to the USP22 promoter (30-32). THP may decrease PKA activity and phosphorylation of CREB-1 to reduce CREB-1 binding to the USP22 promoter, leading to a decreased expression level of USP22 in HeLa cells. Consequently, the downregulated expression of USP22 promoted the apoptosis of HeLa cells. Therefore, the novel results of the present study may be used to elucidate the underlying mechanism of THP-mediated inhibition of USP22 expression in HeLa cells.

In conclusion, the present study indicated that THP induced apoptosis of HeLa cells and decreased the expression of USP22 in dose- and time-dependent manner. THP significantly reduced the USP22 promoter activity, dependent on the binding sequence of CREB, which was not affected by CREB-1 over-expression. Furthermore, THP significantly inhibited the phosphorylation of CREB-1 at ser133 and its binding to the USP22 promoter. Therefore, the novel results of the current study may be used to elucidate the molecular mechanisms underlying the pharmacological pro-apoptotic effect of THP in tumor cells.

\section{Acknowledgements}

Not applicable.

\section{Funding}

The present study was supported by the grants from the National Nature Science Foundation of China (grant nos. 81460172 and 81860165), Natural Science Foundation of Jiangxi, China (grant no. 20151BAB205056) and the Visiting Scholar Special Funding of Jiangxi Association for Science and Technology (grant no. 2016).

\section{Availability of data and materials}

The datasets used and/or analyzed during the current study are available from the corresponding author on reasonable request.

\section{Authors' contributions}

JX and TW designed the study and analyzed the data. XZ and LG performed the western blotting and reverse transcription-quantitative polymerase chain reaction. JL performed cell culture. XX performed the chromatin immunoprecipitation. All authors interpreted the results, and produced and approved the final manuscript.

\section{Ethics approval and consent to participate}

Not applicable.

\section{Patient consent for publication}

Not applicable.

\section{Competing interests}

The authors declare that they have no competing interests.

\section{References}

1. Shinozaki T, Watanabe H, Yanagawa T, Shirakura K and Takagishi K: Pirarubicin-based versus doxorubicin-based osteosarcoma chemotherapy. Ann Pharmacother 36: 996-999, 2002.

2. Li JJ, Di GH, Tang LC, Yu KD, Hu Z, Liu GY, Lu JS, Wu J, Han QX, Shen ZZ and Shao ZM: Adjuvant therapy of breast cancer with pirarubicin versus epirubicin in combination with cyclophosphamide and 5-fluorouracil. Breast J 17: 657-660, 2011.

3. Zou HY, Wu HL, Zhang Y, Li SF, Nie JF, Fu HY and Yu RQ: Studying the interaction of pirarubicin with DNA and determining pirarubicin in human urine samples: Combining excitation-emission fluorescence matrices with second-order calibration methods. J Fluoresc 19: 955-966, 2009.

4. Liu SY, Song SX, Lin L and Liu X: Molecular mechanism of cell apoptosis by paclitaxel and pirarubicin in a human osteosarcoma cell line. Chemotherapy 56: 101-107, 2010.

5. Zheng SE, Xiong S, Lin F, Qiao GL, Feng T, Shen Z, Min DL, Zhang CL and Yao Y: Pirarubicin inhibits multidrug-resistant osteosarcoma cell proliferation through induction of $\mathrm{G} 2 / \mathrm{M}$ phase cell cycle arrest. Acta Pharmacol Sin 33: 832-838, 2012.

6. Wu Y, Ni Z, Yan X, Dai X, Hu C, Zheng Y, He F and Lian J: Targeting the MIR34C-5p-ATG4B-autophagy axis enhances the sensitivity of cervical cancer cells to pirarubicin. Autophagy 12: 1105-1117, 2016.

7. Lee HJ, Kim MS, Shin JM, Park TJ, Chung HM and Baek KH: The expression patterns of deubiquitinating enzymes, USP22 and Usp22. Gene Expr Patterns 6: 277-284, 2006.

8. Zhao Y, Lang G, Ito S, Bonnet J, Metzger E, Sawatsubashi S, Suzuki E, Le Guezennec X, Stunnenberg HG, Krasnov A, et al: A TFTC/STAGA module mediates histone $\mathrm{H} 2 \mathrm{~A}$ and $\mathrm{H} 2 \mathrm{~B}$ deubiquitination, coactivates nuclear receptors, and counteracts heterochromatin silencing. Mol Cell 29: 92-101, 2008.

9. Zhang XY, Pfeiffer HK, Thorne AW and McMahon SB: USP22, an hSAGA subunit and potential cancer stem cell marker, reverses the polycomb-catalyzed ubiquitylation of histone $\mathrm{H} 2 \mathrm{~A}$. Cell Cycle 7: 1522-1524, 2008.

10. Zhang XY, Varthi M, Sykes SM, Phillips C, Warzecha C, Zhu W, Wyce A, Thorne AW, Berger SL and McMahon SB: The putative cancer stem cell marker USP22 is a subunit of the human SAGA complex required for activated transcription and cell-cycle progression. Mol Cell 29: 102-111, 2008.

11. Xiao H, Tian Y, Yang Y, Hu F, Xie X, Mei J and Ding F: USP22 acts as an oncogene by regulating the stability of cyclooxygenase- 2 in non-small cell lung cancer. Biochem Biophys Res Commun 460: 703-708, 2015.

12. Lin Z, Yang H, Kong Q, Li J, Lee SM, Gao B, Dong H, Wei J, Song J, Zhang DD and Fang D: USP22 antagonizes p53 transcriptional activation by deubiquitinating Sirt1 to suppress cell apoptosis and is required for mouse embryonic development. Mol Cell 46: 484-494, 2012.

13. Atanassov BS and Dent SY: USP22 regulates cell proliferation by deubiquitinating the transcriptional regulator FBP1. EMBO Rep 12: 924-930, 2011.

14. Lin Z, Tan C, Qiu Q, Kong S, Yang H, Zhao F, Liu Z, Li J, Kong Q, Gao B, et al: Ubiquitin-specific protease 22 is a deubiquitinase of CCNB1. Cell Discov 1: pii: 15028, 2015.

15. Hu J, Yang D, Zhang H, Liu W, Zhao Y, Lu H, Meng Q, Pang H, Chen X, Liu Y and Cai L: USP22 promotes tumor progression and induces epithelial-mesenchymal transition in lung adenocarcinoma. Lung Cancer 88: 239-245, 2015.

16. Li Y, Yang Y, Li J, Liu H, Chen F, Li B, Cui B and Liu Y: USP22 drives colorectal cancer invasion and metastasis via epithelial-mesenchymal transition by activating AP4. Oncotarget 8 : 32683-32695, 2017.

17. Glinsky GV: Death-from-cancer signatures and stem cell contribution to metastatic cancer. Cell Cycle 4: 1171-1175, 2005.

18. Xiong J, Che X, Li X, Yu H, Gong Z and Li W: Cloning and characterization of the human USP22 gene promoter. PLoS One 7: e52716, 2012.

19. Xiong J, Gong Z, Zhou X, Liu J, Jiang HE, Wu P and Li W: p38 mitogen-activated protein kinase inhibits USP22 transcription in HeLa cells. Biomed Rep 3: 461-467, 2015. 
20. Xiong J, Xu X, Zhou X, Liu J, Gong Z, Wu P and Li W: USP22 transcriptional activity is negatively regulated by the histone deacetylase inhibitor trichostatin A. Mol Med Rep 10: 3343-3347, 2014.

21. Livak KJ and Schmittgen TD: Analysis of relative gene expression data using real-time quantitative PCR and the 2(-Delta Delta C(T)) method. Methods 25: 402-408, 2001.

22. Ling S, Li J, Shan Q, Dai H, Lu D, Wen X, Song P, Xie H, Zhou L, Liu J, et al: USP22 mediates the multidrug resistance of hepatocellular carcinoma via the SIRT1/AKT/MRP1 signaling pathway. Mol Oncol 11: 682-695, 2017

23. Xie Z, Liu D, Liu S, Calderon L, Zhao G, Turk J and Guo Z: Identification of a cAMP-response element in the regulator of G-protein signaling-2 (RGS2) promoter as a key cis-regulatory element for RGS2 transcriptional regulation by angiotensin II in cultured vascular smooth muscles. J Biol Chem 286: 44646-44658, 2011.

24. Xiong J, Zhou X, Gong Z, Wang T, Zhang C, Xu X, Liu J and Li W: PKA/CREB regulates the constitutive promoter activity of the USP22 gene. Oncol Rep 33: 1505-1511, 2015.

25. He X, Li J, Guo W, Liu W, Yu J, Song W, Dong L, Wang F, Yu S, Zheng Y, et al: Targeting the microRNA-21/AP1 axis by 5 -fluorouracil and pirarubicin in human hepatocellular carcinoma. Oncotarget 6: 2302-2314, 2015.

26. Zhou D, Liu P, Sun DW, Chen ZJ, Hu J, Peng SM and Liu YL: USP22 down-regulation facilitates human retinoblastoma cell aging and apoptosis via inhibiting TERT/P53 pathway. Eur Rev Med Pharmacol Sci 21: 2785-2792, 2017.
27. Tang B, Tang F, Li B, Yuan S, Xu Q, Tomlinson S, Jin J, Hu W and He S: High USP22 expression indicates poor prognosis in hepatocellular carcinoma. Oncotarget 6: 12654-12667, 2015.

28. Ovaa H, Kessler BM, Rolén U, Galardy PJ, Ploegh HL and Masucci MG: Activity-based ubiquitin-specific protease (USP) profiling of virus-infected and malignant human cells. Proc Nat Acad Sci USA 101: 2253-2258, 2004.

29. Li K, Chen X, Liu C, Gu P, Li Z, Wu S, Xu K, Lin T and Huang J: Pirarubicin induces an autophagic cytoprotective response through suppression of the mammalian target of rapamycin signaling pathway in human bladder cancer cells. Biochem Biophys Res Commun 460: 380-385, 2015.

30. Montminy MR, Sevarino KA, Wagner JA, Mandel G and Goodman RH: Identification of a cyclic-AMP-responsive element within the rat somatostatin gene. Proc Natl Acad Sci USA 83: 6682-6686, 1986.

31. Vo N and Goodman RH: CREB-binding protein and p300 in transcriptional regulation. J Biol Chem 276: 13505-13508, 2001.

32. Delghandi MP, Johannessen M and Moens U: The cAMP signalling pathway activates CREB through PKA, p38 and MSK1 in NIH 3 T3 cells. Cell Signal 17: 1343-1351, 2005. 